Journal of Thermal Engineering

Yildiz Technical University Press, Istanbul, Turkey

Vol. 1, Special Issue 3, No. 6, pp. 402-407, May, 2015.
http://eds.yildiz.edu.tr/journal-of-thermal-engineering/Articles

Manuscript Received January 20, 2015; Accepted February 07, 2015

This paper was recommended for publication in revised form by Editor in Chief Ahmet Selim Dalkilic

\title{
Thermoelectric Properties of Bismuth Telluride Filled Silicone
}

\author{
Bruce Y. Decker \\ Department of Mechanical Engineering, \\ California State Polytechnic University, Pomona, \\ 3801 W Temple Avenue, Pomona, CA 91768
}

\author{
*Yong X. Gan \\ Department of Mechanical Engineering, \\ California State Polytechnic University, Pomona, \\ 3801 W Temple Avenue, Pomona, CA 91768
}

\author{
Sinclair Calderon \\ Department of Mechanical Engineering, \\ California State Polytechnic University, Pomona, \\ 3801 W Temple Avenue, Pomona, CA 91768
}

Keywords: Thermoelectric materials; bismuth telluride; composite material

* Corresponding author: Y.X. Gan, Phone: +1-909-869-2388, Fax: +1-909-869-4341

E-mail address:yxgan@csupomona.edu

\section{ABSTRACT}

Bismuth telluride filled silicone rubber composite was extruded into millimeter-sized wires using electrospinning. The composite wires were tested in view of the electrical resistance and Seebeck coefficient. The highest electrical resistance measured is $2.9^{*} 10^{10} \mathrm{ohms}$. The composite material exhibited high Seebeck effect because silicone rubber exhibits low thermal conductivity as a result of increased phonon scatters. Moreover, compared with the bulk reference material, the thermoelectric property of bismuth telluride is notably enhanced. Due to the inherent flexibility of silicone rubber and thermoelectric property of bismuth telluride, it is possible for making a flexible thermoelectric material for alternative energy applications.

\section{INTRODUCTION}

Thermoelectric materials have the capability to convert waste heat energy into useful electric energy. In recent years, much research has been done to observe such phenomenon. There are limitations associated with thermoelectric composite materials. It is a compromise between thermal conductivity and electrical conductivity of the thermoelectric material. To optimize the Seebeck effect, thermal conductivity should be reduced whereas electrical conductivity is supposed to be enhanced. Furthermore, high performance thermoelectric materials can be applied to recover waste heat from industry and vehicle tail pipes. The internal combustion process of an engine wastes a huge portion of the energy as waste heat, thermoelectric materials can be implemented to recover waste heat and higher efficiency is possible to achieve without any modification on the engine.

As a compound of tellurium and bismuth, bismuth telluride exhibits excellent thermoelectric property when it is alloyed with other elements such as selenium or antimony. Bismuth telluride has been used for the construction of thermoelectric modules due to its high thermoelectric figure of merit [1]. Bulk bismuth telluride alloy is one of the most widely used commercial thermoelectric materials. It has a maximum ZT close to unity at room temperature [2]. The thermoelectric figure of merit in a dimensionless form, ZT, has increased from 0.5 to greater than one in today's standard [1]. $\mathrm{Z}$ is referred as the power factor. $\mathrm{T}$ is the absolute temperature. ZT is a function of Seebeck coefficient $(\mathrm{S})$, electrical conductivity $(\sigma)$, thermal conductivity $(\kappa)$, and absolute temperature (T). The equation is given by $\mathrm{ZT}=\mathrm{S}^{2} \sigma \mathrm{T} /$ $\kappa$. Like described in the previous paragraph, a good thermoelectric material should have a high $\sigma$ and a low $\kappa$. An excellent thermoelectric material should have a low thermal conductivity value. In general, increased scattering of heat carriers at interfaces lowers $\kappa$ value. Changes in charge carrier density near the Fermi level could increase $\sigma$ and S [3]. Fermi level is the top collection of electron energy levels at absolute zero temperature and the probability of occupation by electrons is one half. 
$\mathrm{Bi}_{2} \mathrm{Te}_{2}$ based solid solutions usually have $\mathrm{ZT}$ values close to 1. They are manufactured by unidirectional crystal growth methods such as Bridgeman or zone melting technique that would lead to the preferred crystalline orientation [4]. Nevertheless, the high degree of texture is associated with the weak van der Waals bonding between $\mathrm{Te}^{(1)}$ and $\mathrm{Te}^{(1)}$ layers of their quasi-layered crystalline structure which results in poor mechanical properties [4].

Lattice thermal conductivity is inherently related to phonon transport [5]. In semiconductors and insulators, heat is transferred and quantified by vibrations in the crystal lattice. Inside a material, high frequencies are associated with heat while low frequencies correlate to sound. Thermal conductivity $\kappa$ comes from phonons traveling through the crystal lattice and electrons and is consisted of phonon contribution and carrier contribution which are related to the electrical conductivity $\sigma$ according to the Wiedemann-Franz law [6]. The law describes raising the temperature would result in increasing in the thermal conductivity while decreasing the electrical conductivity. Often times, the electrical conductivity is easy to be measured. However, the thermal conductivity is estimated by the Wiedemann-Franz (WF) law since the ratio of heat and charge transport by electrons is constant [7]. The total thermal conductivity is contributed by phonons and electrons. As a consequence, the thermal conductivity caused by phonons and electrons are tested separately [7]. The electron thermal conductivity is calculated by $\chi_{\mathrm{e}}=\mathrm{L} \sigma \mathrm{T}$ where the constant of proportionality is the Lorenz number. By definition, the total thermal conductivity is consisted of phonon thermal conductivity and electron thermal conductivity. Thus, the equation is given by $\chi_{\text {ph }}=\chi_{\text {Total }}-\chi_{\mathrm{e}}[7]$.

Bismuth telluride $\left(\mathrm{Bi}_{2} \mathrm{Te}_{3}\right)$ is also a low temperature thermoelectric material and is applied in thermoelectric generators and coolers [8]. The electrical and thermal conductivities of bismuth telluride are anisotropic which means it has different properties in different directions but Seebeck coefficient does not depend on orientation in the extrinsic or onecarrier regime [1]. The $\mathrm{Bi}_{2} \mathrm{Te}_{3-\mathrm{y}} \mathrm{Se}_{\mathrm{y}}$ single crystal bulk is a lamella structure and van der Waals bonding force between $\mathrm{Te}^{(1)}-\mathrm{Te}^{(1)}$ causes the easy cleavage along the planes perpendicular to the caxis. In contrast, n-type $\mathrm{Bi}_{2} \mathrm{Te}_{3-y} \mathrm{Se}_{\mathrm{y}}$ single crystal solid solutions exhibits strong anisotropic property [9].

The crystal structure of $\mathrm{Bi}_{2} \mathrm{Te}_{3}$ is rhombohedral with the space group $D_{3 d}^{5}(\mathrm{R} \overline{3} \mathrm{~m})$. It has five atoms in the trigonal unit cell [10]. As a semiconductor, it has a $0.150 \mathrm{eV}$ energy gap [11]. The energy gap is referred to the difference between the valence band and the conduction band. As a narrow-band semiconductor compound, it is crystallized in the structure of tetradymite $\mathrm{Bi}_{2} \mathrm{Te}_{2} \mathrm{~S}$. Teradymite also has rhombohedral symmetry (space group $D_{3 d}^{5}=\mathrm{R} \overline{3} \mathrm{~m}$, structure type C33) [12]. The rhombohedral unit cell contain one $\mathrm{Bi}_{2} \mathrm{Te}_{3}$ molecule but the structure is usually referred as a hexagonal unit cell consisting of 15 layers of three five layer packets. The reason for referring it as the hexagonal unit cell is that it is convenient to rearrange it into the hexagonal unit cell build up by three formula units [13]. For instance, they are quintets $\mathrm{Te}(1)-\mathrm{Bi}-\mathrm{Te}(2)-\mathrm{Bi}-\mathrm{Te}(1)$-alternating along the hexagonal axis [12]. The closest neighbors of $\mathrm{Te}(1)$ are three $\mathrm{Te}(1)$ atoms and three $\mathrm{Bi}$ atoms. In contrast, $\mathrm{Te}(2)$ has six $\mathrm{Bi}$ atoms that are the closest. $\mathrm{Te}_{2}-\mathrm{Bi}$ bond is inherently covalent, and the Te1-Bi bond is mixed covalent and ionic [14]. The hexagonal cell contains 15 atoms grouped in the 3 quintuple layers as described from above. The rhombohedral unit-cell parameters are $a_{R}=10.473 \AA$ and $\theta_{R}=24.159 \AA$ at $293 \mathrm{~K}$. In addition, the hexagonal unit-cell parameters are $\mathrm{a}=10.473 \AA$ and $\mathrm{c}=30.487$ $\AA$ [15]. $\mathrm{Te}(1)$ and $\mathrm{Te}(2)$ are two different crystal kinds of tellurium atoms. For Bismuth Telluride, the hexagonal lattice parameters are $a \frac{h e x}{B i T e}=4.384 \AA, \mathrm{c} \frac{h e x}{B i T e}=30.487 \AA$. For Be-Te2, the closest interatomic distances between the individual monolayers inside the quintuple blocks are $3.25 \AA$ [12]. However, the bond length of Tel-Bi is $3.07 \AA$, which is shorter than the bond of $\mathrm{Bi}-\mathrm{Te} 2$ [15].

Polymers such as silicone rubber provides flexibility to the composite, but due to its insulating behavior, it does hinder Seebeck effect [16]. However, the silicone rubber matrix is filled with semiconductor bismuth telluride. The presence of silicone rubber matrix can increase phonon scatters and hence reduce thermal conductivity. Since silicon rubber possesses high heat resistance and thermal stability, it can be implemented in a large temperature gradient environment for thermoelectric applications. Silicone rubber compounds are made conductive by adding conductive filler such as carbon, metal powders, carbon fibers, carbon nanotubes and graphite [17]. Despite the conductivity of carbon, it can potentially slow down the curing of rubber when it is used in high concentration. There is a drawback when adding fillers in the silicone rubber. The strength and flexibility of the composites will be sacrificed if fillers are added in a large quantity. Another process that will enhance the conductivity of silicon rubber composites is heat treatment [18].

\section{MATERIAL AND EXPERIMENTAL METHODS}

0.2 gram of bismuth telluride was mixed with 1.0 grams of silicone rubber. The total weight was added to 1.2 grams, and 0.12 grams of the mixture was then transported to the syringe, which amounted to $10 \%$ of the bismuth telluride powder. In addition, the powder used in this experiment had $99.98 \%$ purity. The syringe needle used was a reusable stainless steel dispensing needle from McMaster-Carr with a part number of 6710A41. The inside diameter of the syringe needle was $2.15 \mathrm{~mm}$ and the outside diameter was $2.769 \mathrm{~mm}$. The length of the syringe needle was $101.6 \mathrm{~mm}$. As shown in Fig. 1, a syringe pump was instructed to pump the mixture at a rate of $0.1 \mathrm{~mL}$ per minute. In order to achieve electrospinning, a DC power supply was implemented to deliver up to $30 \mathrm{kV}$ of voltage to the syringe tip. However, severe discharging of electricity was observed when the voltage was set at $18 \mathrm{kV}$. Processed wires exhibited whipping rotations when the voltage was set at $16 \mathrm{kV}$. Then, the ground lead from the DC power supply was connected to the metal plate as shown in Fig. 1. Furthermore, the rectangular metal plate functioned as a grounded collector or a counter electrode. Due to the high-voltage electric field, the bismuth telluride and silicone 
rubber mixture was stretched and extruded under electrostatic attraction and surface tension.

For the Seebeck coefficient measurements, a Talboy electrical heating plate was used to produce temperature difference within the processed material. The rapid changes in temperature were measured by an infrared thermometer. To accommodate experimental accuracy and temperature gradient, one end of the sample was contacted to the Talboys hot plate, and the other end of the sample was left at the ambient temperature which was close to $25^{\circ} \mathrm{C}$. Two ends of bismuth telluride/silicone composite $\mathrm{rod} /$ wire specimens were wrapped by aluminum foils to ensure optimal electrical conductivity. The aluminum foil at each end served the purpose of creating electrical conducting path for the electrical resistance and the Seebeck coefficient measurements. A CHI 600E electrochemical analyzer was used to record the current and electrode potential. The resistivity and Seebeck coefficient were calculated using the recorded data from the measurements.

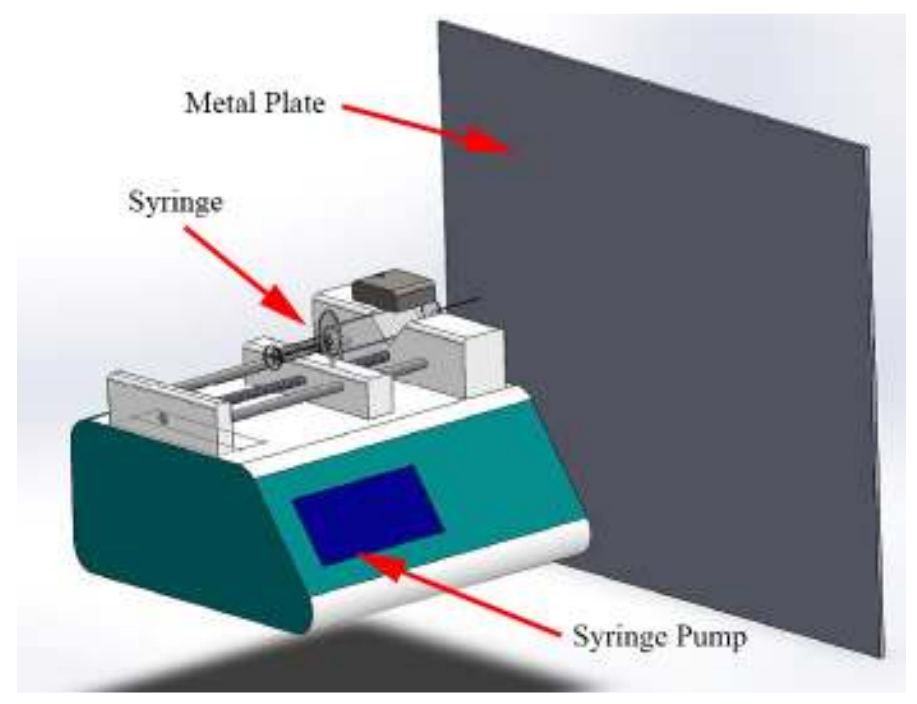

Figure 1: Schematic of a syringe connected to a power supply delivering DC voltage

\section{DATA REDUCTION}

Although thermal conductivity of bismuth telluride filled with silicone rubber matrix was not tested, the Wiedemann-Law can be used to relate the electrical conductivity to the electrical component of the thermal conductivity $\chi^{\mathrm{e}} / \sigma \mathrm{T}=$ constant $=$ Lorenz number, where $\chi^{\mathrm{e}}$ is electron thermal, and $\sigma$ stands for electrical conductivity [20]. The Wiedemann-Law equation is obtained by combining the first and second equations listed below. By combining electron charge and thermal conductivity derived from the Boltzmann transport equation, the equation becomes

$$
\frac{k_{e}}{\sigma}=\frac{\frac{1}{3} v_{F}^{2} \tau_{F} C_{e}}{\frac{e^{2}}{m} n \tau_{F}}=\frac{m C_{e} v_{F}^{2}}{3 n e^{2}}
$$

Furthermore, $\mathrm{C}_{\mathrm{e}}$ is the electron specific heat. $v_{F}$ is the Fermi velocity. $\tau_{F}$ is the scattering mean free time of Fermi electrons, and $\mathrm{n}$ is the number density. $\mathrm{m}$ stands for mass. $\sigma$ is as known as electrical conductivity, and $\mathrm{k}_{\mathrm{e}}$ is the thermal conductivity. $\mathrm{e}$ is the electron charge [21] [22]. By deriving from the thermal conductivity transport equation, the electron specific heat $\mathrm{C}_{\mathrm{e}}$ is obtained by

$$
\mathrm{C}_{\mathrm{e}}=\frac{\frac{1}{2} \pi^{2} n k_{B} T}{T_{F}}=\frac{\frac{1}{2} \pi^{2} n k_{B} T}{\frac{m v_{F}^{2}}{2 k_{B}}}=\frac{\pi^{2} n k_{B}^{2} T}{m v_{F}^{2}}
$$

where $\mathrm{k}_{\mathrm{B}}$ is the Boltzmann constant that is equal to $1.3807 * 10^{-23}$ $\mathrm{m}^{2 *} \mathrm{~kg}^{*} \mathrm{~s}^{-2 *} \mathrm{~K}^{-1}$, and $\mathrm{T}$ stands for temperature. Likewise, $\mathrm{T}_{\mathrm{F}}$ is the Fermi temperature.

Thus, the ratio between thermal and electrical conductivity is described by

$$
\frac{k_{e}}{\sigma}=\frac{m v_{F}^{2}}{3 n e^{2}} \frac{\pi^{2} n k_{B}^{2} T}{m v_{F}^{2}}=\frac{\pi^{2} k_{B}^{2} T}{3 e^{2}}
$$

The proportionality constant, Lorenz number is defined by

$$
\mathrm{L}=\frac{\pi^{2} k_{B}^{2}}{3 e^{2}}=2.45 \times 10^{-8} \frac{W \Omega}{K^{2}}
$$

Lastly, the Wiedemann-Franz law is defined by

$$
\frac{k_{e}}{\sigma}=\mathrm{LT}
$$

Thus, the ratio between thermal conductivity and electrical conductivity is proportional to the composite material temperature.

\section{RESULTS AND DISCUSSIONS}

Polymer such as silicone rubber possesses low thermal conductivity and high electrical resistance. Pure polymeric materials usually exhibit a low conductivity that is less than 0.2 $\mathrm{W} /(\mathrm{m} \cdot \mathrm{K})$ [19]. In this experiment, silicone matrix was used as an insulator. Two separate sets of experiments were performed to test for electrical resistivity and Seebeck coefficient. It was expected that the presence of the silicone rubber matrix would increase phonon scatters and hence reduce thermal conductivity. Moreover, the figure of merit would increase due to low thermal conductivity.

Two composites of the bismuth telluride filled with silicone wires were tested for resistivity and Seebeck coefficient. The first sample was tested for resistance and I-V relation in two experimental trials as shown in Fig. 2 and Fig. 3. The voltammetry method used to test for resistance was linear sweep voltammetry which indicates voltage was swept linearly with time between the working electrodes. The final voltage was set at $10 \mathrm{~V}$, and the scan rate was adjusted at $0.1 \mathrm{~V}$ per second (Fig.2 \& 3). The same sample was tested two additional times for resistance to confirm accurate results. The test result showed no significant discrepancies in two consecutive experiment trials. In 
addition, by analyzing several experimental trials, silicone rubber inherently exhibits high electrical resistance and the experiment showed that the silicone rubber control sample had an average resistance of $1.785^{*} 10^{10} \mathrm{ohms}$. The highest resistance tested in the first sample was $2.9 * 10^{10}$ ohms since bismuth telluride exhibited topological insulating behavior and possessed overlapping conduction and valence bands, with a single nondegenerate surface-state band exhibiting a Dirac cone structure [23]. Unlike adding conductive fillers such as carbon and metal powders, filling bismuth telluride in the silicone matrix will increase the overall electrical resistance of the composite material. Since this was an experiment conducted to determine the Seebeck coefficient and electrical resistance of the composite material, errors and uncertainties were inevitable, and multiple trials of the experiment were performed to testify accuracy. Errors could be resulted by false readings of the electrochemical analyzer CHI 600. When testing for Seebeck coefficient, the composite material was heated using a Talboy heating plate. Due to the fact that there was a time delay when measuring the temperature difference between opposite ends of the composite material, errors could be resulted in the Seebeck coefficient data.

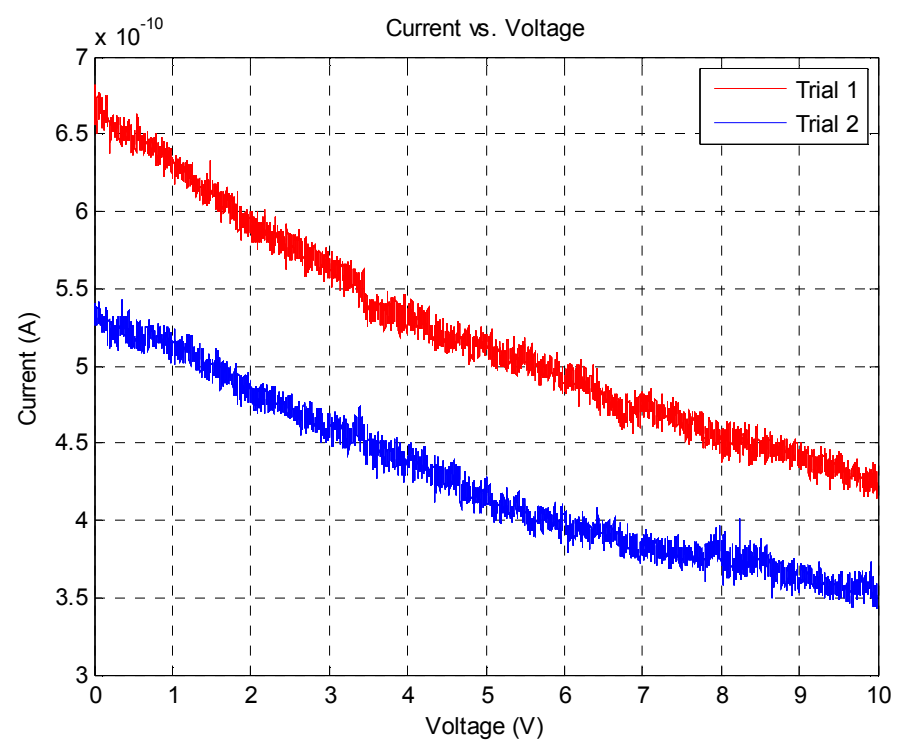

Figure 2: Current responses of the first composite under the supplied voltage with an increment of $0.001 \mathrm{~V}$ from 0 to $10 \mathrm{~V}$

In order to reduce thermal conductivity and to increase the figure of merit, increase in electrical has to be incurred as a result of using silicone rubber matrix. Fig. 4 presented Seebeck coefficient of the material with varying hot end temperatures at $313 \mathrm{~K}$ and $321 \mathrm{~K}$. As the sample was heated on the Talboy electrical heating plate, the $\mathrm{CHI}$ electrochemical workstation took voltage data vs. time. The temperature gradients were recorded simultaneously along with the voltage data. The output voltage in the sample was measured incrementally every 10 seconds within the time span of 150 seconds in the open circuit mode. The average Seebeck value at the hot end temperature of $321 \mathrm{~K}$ was $0.01406 \mathrm{~V} / \mathrm{K}$, and an average Seebeck value of 0.029 $\mathrm{V} / \mathrm{K}$ at $313 \mathrm{~K}$. The measured values of bismuth telluride filled with silicone matrix are significantly higher than the bulk reference value. It is apparent that the presence of bismuth telluride in the silicone rubber matrix can effectively enhance the thermoelectric property.

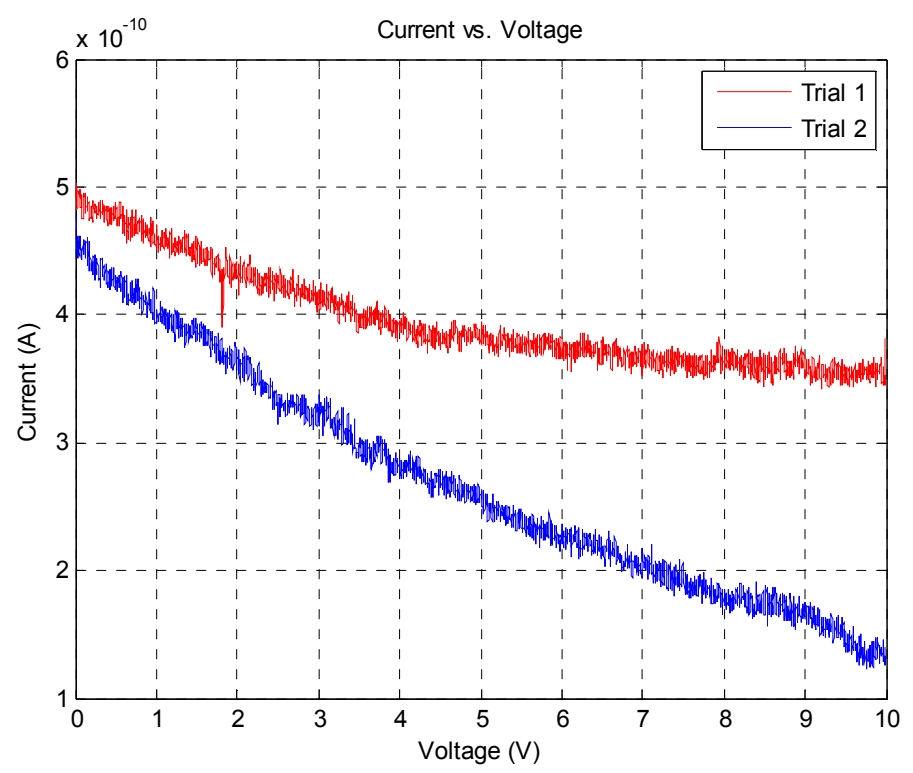

Figure 3: Current responses of the second composite under the supplied voltage with an increment of $0.001 \mathrm{~V}$ from 0 to $10 \mathrm{~V}$



Figure 4: Seebeck Coefficient measured at the hot end temperatures of $313 \mathrm{~K}$ and $321 \mathrm{~K}$ 


\section{CONCLUSION}

In summary, the experiment investigated thermoelectric property and electrical resistance of bismuth telluride filled silicone matrix through the electrospinning process. The highest electrical resistance measured in this experiment is $2.9^{*} 10^{10}$ ohms. The average measured resistance is $1.5325^{*} 10^{10} \mathrm{ohms}$. Although electrical resistance is increased by using silicone rubber matrix, thermal conductivity is reduced as a result of an increase in phonon scatters. Hence, there is a compromise between the electrical resistance and the thermal conductivity when choosing silicone rubber as the matrix. As a result, the thermoelectric property of the composite material is increased significantly compared with the bulk reference material.

\section{ACKNOWLEDGMENTS}

This work is supported by National Science Foundation under Grant Number CMMI 1333044.

\section{REFERENCES}

[1] Goldsmid HJ, "Bismuth Telluride and Its Alloys as Materials for Thermoelectric Generation”, Materials, 7, 25772592 (2014)

[2] Mavrokefalos A, Moore AL, Pettes MT, Shi L, Wang W, Li $\mathrm{XG}$, "Thermoelectric and structural characterizations of individual electrodeposited bismuth telluride nanowires", Journal of Applied Physics, 105, 104318 (2009)

[3] Purkayastha A, Kim S, Gandhi DD, Ganesan PG, BorcaTasciuc T, Ramanath G, "Molecularly Protected Bismuth Telluride Nanoparticles: Microemulsion Synthesis and Thermoelectric Transport Properties", Advanced Materials, 18, 2958-2963 (2006)

[4] Xu ZJ, Hu LP, Ying PJ, Zhao XB, Zhu TJ, “Enhanced thermoelectric and mechanical properties of zone melted $p$-type $(\mathrm{Bi}, \mathrm{Sb})_{2} \mathrm{Te}_{3}$ thermoelectric materials by hot deformation", Acta Materialia, 84, 385-392 (2015)

[5] Wang YG, Qiu B, McGaughey AJH, Ruan XL, Xu XF, "Mode-Wise Thermal Conductivity of Bismuth Telluride", Journal of Heat Transfer, 135, 091102 (2013)

[6] Pinisetty D, Devireddy RV, "Thermal conductivity of semiconductor (bismuth-telluride)-semimetal (antimony) superlattice nanostructures", Acta Materialia, 58, 570-576 (2010)

[7] Wang HD, Liu JH, Zhang X, Takahasi K, "Breakdown of Wiedemann-Franz law in individual suspended polycrystalline gold nanofilms down to $3 \mathrm{~K}$ ", International Journal of Heat and Mass Transfer, 66, 585-591 (2013)

[8] Dheepa J, Sathyamoorthy R, Velumani S, Subbarayan A, Natarajan K, Sebastian PJ, "Electrical resistivity of thermally evaporated bismuth telluride thin films", Solar Energy

Materials \& Solar Cells, 81, 305-312 (2004)

[9] Yan X, Poudel B, Ma Y, Liu WS, Joshi G, Wang H, Lan YC, Wang DZ, Chen G, Ren ZF, "Experimental Studies on Anisotropic Thermoelectric Properties and Structures of n-Type $\mathrm{Bi}_{2} \mathrm{Te}_{2.7} \mathrm{Se}_{0.3}$ ", Nano Letters, 10, 3373-3378 (2010)

[10] Mishra SK, Satpathy S, Jepsen O, "Electronic structure and thermoelectric properties of bismuth telluride and bismuth selenide", Journal of Physics: Condensed Matter, 9, 461-470 (1997)

[11] Goncalves LM, Couto C, Alpuim P, Rolo AG, Völklein F, Correia JH, "Optimization of thermoelectric properties on $\mathrm{Bi}_{2} \mathrm{Te}_{3}$ thin films deposited by thermal co-evaporation", Thin Solid Films, 518, 2816-2821 (2010)

[12] Pavlova LM, Shtern YI, Mironov RE, "Thermal Expansion of Bismuth Telluride”, High Temperature, 49, 369-379 (2011)

[13] Yavorsky BY, Hinsche NF, Mertig I, Zahn P, "Electronic structure and transport anisotropy of $\mathrm{Bi}_{2} \mathrm{Te}_{3}$ and $\mathrm{Sb}_{2} \mathrm{Te}_{3}$ ", Physical Review B, 84, 165208 (2011)

[14] Tong Y, Yi FJ, Liu LS, Zhai PC, Zhang QJ, "Molecular dynamics study on thermo-mechanical properties of bismuth telluride bulk", Computational Materials Science, 48, 343-348 (2010)

[15] Huang BL, Kaviany M, "Ab initio and molecular dynamics predictions for electron and phonon transport in bismuth telluride", Physical Review B, 77, 125209 (2008)

[16] Kowalik D, Chung DDL, "Carbon Black Filled Silicone as a Compliant Thermoelectric Material”, Journal of Reinforced Plastics and Composites, 21, 1587-1590 (2002)

[17] Joshi AM, Athawale AA, "Electrically Conductive Silicone/Organic Polymer Composites”, Silicon, 6, 199-206 (2014)

[18] Hu SF, Li H, Chen XX, Zhang C, Liu ZF, “The Electrical Conductive Effect of Nickel-coated Graphite/Two-component Silicone-rubber Sealant", Journal of Wuhan University of Technology-Materials Science Edition, 28, 429-436 (2013)

[19] Cheng JP, Liu T, Zhang J, Wang BB, Ying J, Liu F, Zhang $\mathrm{XB}$, "Influence of phase and morphology on thermal conductivity of alumina particle/silicone rubber composites", Applied Physics A, 117, 1985-1992 (2014)

[20] Vekilov YK, Isaev EI, Johansson B, "Does the Wiedemann-Franz law work for quasicrystals?", Physics Letters A, 352, 524-525 (2006) 
[21] Westly N, "Electronic Transport in Thermoelectric Bismuth Telluride", University of New Orleans Theses and Dissertations, Paper 1539 (2012)

[22] Arabshahi H, Sarlak F, "A New Study on Calculation of Electron Transport Characteristics in Semiconductor
Materials", International Archive of Applied Sciences and Technology, 1, 71-80 (2010)

[23] Kioupakis E, Tiago ML, Louie SG, "Quasiparticle electronic structure of bismuth telluride in the $\mathrm{GW}$ approximation", Physical Review B, 82, 245203 (2010) 